\title{
Finding the Optimum Orientation for PV Systems Matched to the Timing of the Demand Profile
}

\author{
Jubran Alshahrani \\ Institute of Energy and Sustainable \\ Development \\ De Montfort University \\ Leicester, UK \\ Jubran.2050@gmail.com
}

\author{
Peter Boait \\ Institute of Energy and Sustainable \\ Development \\ De Montfort University \\ Leicester, UK
}

\author{
Abdullah Alshahrani \\ Faculty of Engineering \\ University of Nottingham \\ Nottingham, UK
}

\begin{abstract}
Electricity consumption in the Kingdom of Saudi Arabia (KSA) has grown by about $7 \%$ annually in the last two decades due to population and economic growth. The consumption of the residential sector accounts for over $50 \%$ of the total energy generation largely due to the consumption of the buildings' air conditioning. This factor contributes significantly to a situation where peak electricity demand occurs in early afternoon. Thus, this paper presents one of the possible ways of managing electricity peak demand by proposing deployment of PV panels with slope and orientation that are optimized with respect to the timing of the demand profile in order to contribute most effectively to national electricity generation capacity. As a case study, numerical results are presented for Riyadh city in KSA.
\end{abstract}

Keywords-Photovoltaic panel orientation; peak demand profile; air conditioning demand

\section{NOMENCLATURE}

$\varphi$ - Latitude of the location of the PV system

$S$ - Solar radiation power constant, $1.367 \mathrm{~kW} / \mathrm{m}^{2}$

$e_{c}$ - Empirical constant, $e_{c}=0.65$ if $\varphi>45^{\circ}$ or $e_{c}=0.75$ if $\varphi \leq 45^{\circ}$

$B-\mathrm{PV}$ panel elevation angle from the horizontal

$\delta$ - Solar declination angle

$\omega$ - Solar hour angle (noon is $0^{\circ}$ )

$\omega_{s}-$ Solar hour angle for sunrise (and $-\omega_{s}$ for sunset)

$\alpha$-Solar elevation angle

$n$ - Day number in a year

$K$ - Clearness index for a day

$B$ - Solar beam energy on a unit horizontal area for day and location

$D$ - Solar diffuse energy on a unit horizontal area for day and location

$r_{b}$ - Panel angle incident beam radiation factor

$r_{d}$ - Panel angle incident diffuse radiation factor

$G$ - The total solar energy on unit solar panel area over a given day and location and for a given panel angle of elevation

\section{INTRODUCTION}

The electricity market in the Kingdom of Saudi Arabia (KSA) has grown rapidly over the past 20 years by about $7 \%$ annually as shown in Fig.1. The peak demand in the summer has increased by $120 \%$ between 2004 and 2016 (that is, from 28 GW in 2004 to about $62 \mathrm{GW}$ in 2016). This growth is expected to continue annually by at least $6 \%$. This growth is largely driven by population growth, a high demand for air conditioning (AC) during the summer months, and low electricity tariffs [1], [2]. In particular, up to $70 \%$ of the electricity sold is attributed to air conditioning in residential buildings, with summer peak demand nearly twice the winter average [1].

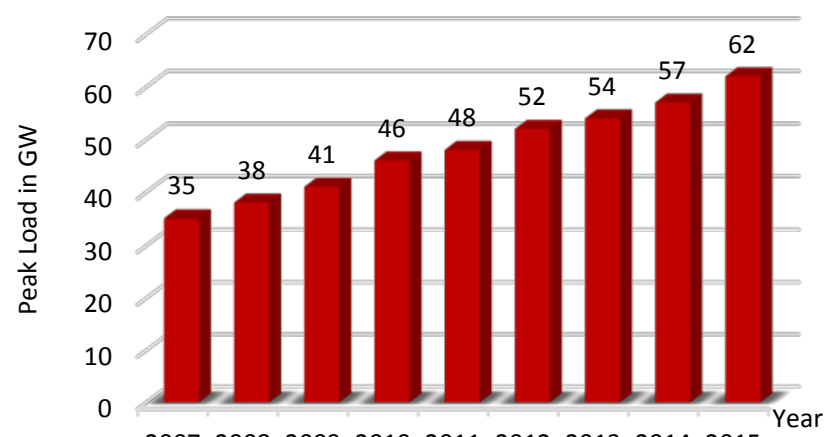

200720082009201020112012201320142015

Fig. 1. The Electricity Peak Load, Saudi Arabia, 2015 [1].

The electricity sector in KSA is faced with great challenges in meeting the increasing electricity consumption demand that is the foundation for social and economic growth. The residential sector consumes over $50 \%$ of KSA's total electricity production - the remaining $50 \%$ being split among industry, commercial sector and governmental agencies. As illustrated in Fig. 2, peak demand for electric power in KSA occurs between 12:00 and 17:00 in summer months (from May to September). This surge in demand is mainly attributed to the loads required by air conditioning systems for cooling [2]. Electricity demand is continuing to outpace growth in supply in recent times, prompting the Saudi Electricity Company (SEC) to limit electricity for some areas during peak demand times. SEC is also requesting some large customers to turn off their supply from 
the grid at certain times and to put their emergency generators in operation during the summer peak period [3].

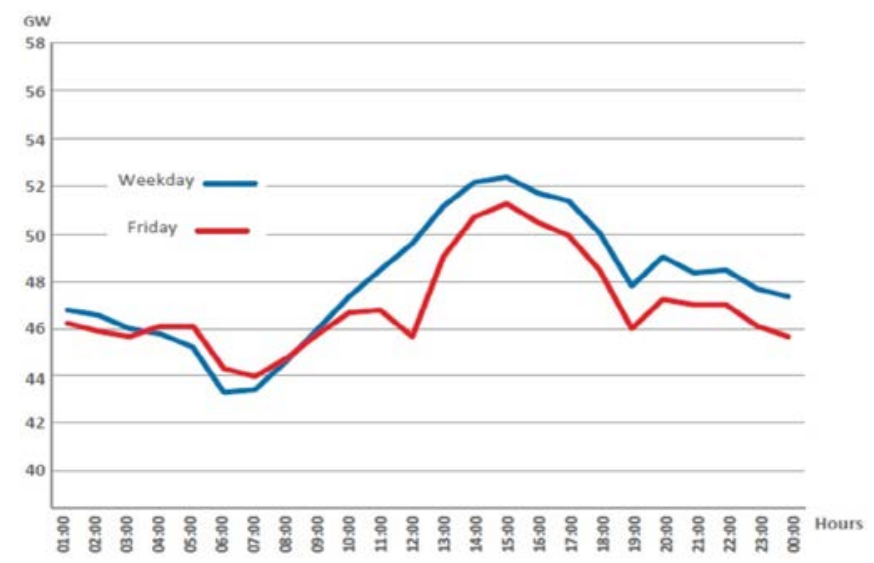

Fig. 2. Typical daily load curve during summer in KSA [2]

In addition, with the aim of meeting the peak demand, the construction of additional power plant or improvements on existing ones is required. In fact, additional generation capacity of 2 to $4 \mathrm{GW}$ is added each year to meet the country's growing electricity demand and, with a projected peak in 2020 of about $60 \mathrm{GW}$, the country would need to add five $1000 \mathrm{MW}$ plants per year to 2020 [4], [5]. The new committed capacity, according to King Abdullah City for Atomic and Renewable Energy (K.A.CARE), will not be sufficient to meet the projected demand. Accordingly, the rapid growth of electricity demand and consumption in KSA is a fundamental problem in need of new solutions. This paper presents one of the workable solutions of tackling this problem by proposing an analysis that enables the deployment of PV panels with slope and orientation that are optimized with respect to the timing of the demand profile in order to satisfy that demand most effectively.

In KSA, due to the availability of solar radiation energy, which is between $2000 \mathrm{kWh} / \mathrm{m}^{2}$ and $2200 \mathrm{kWh} / \mathrm{m}^{2}$ on the average for its annual 3245 sunshine hours per annum, there is enormous potential for using the solar energy [6], [7]. Indeed, photovoltaic technology has proven to be a simple and yet effective means of energy generation in KSA [7].

A review of the literature about PV panel orientation shows that a wide range of optimal tilt angles have been recommended by different authors; moreover, these recommendations are mostly for specific locations, for instance, for Abu Dhabi (UAE) [8], Tabass (Iran) [9], Madinah (Saudi Arabia) [10], and so on. It is obvious that always orienting the PV modules in the optimum angle at every given moment of the day will provide the best performance; however, that is impractical because achieving that will involve the use of tracker systems, which require energy for their operation and are not economic for this application. The algorithm presented in this paper, although demonstrated for the case of the city of Riyadh in Saudi Arabia, is applicable to all other locations by suitable choice of appropriate parameters (such as the location's latitude, clearness of the sky, cloudiness of the climate, etc.). The algorithm finds the optimum tilt and azimuth angle that matches the timing of the peak demand on the load profile

The subsequent sections are arranged as follows: theoretical background (that is, equations governing PV systems) is presented in Section $\Pi$. The algorithm for finding the optimum orientation is given in Section Ш. Numerical results for the case study of Riyadh city are given in Section IV. The discussions on the results of setting the tilt angle to non-optimum values such as to the latitude of the location of the PV system, to the average of optimum tilt angle for five summer months, and to the value for which yearly maximum energy are given in Section IV . The results of the matched and unmatched cases of finding the optimum tilt angles when a PV system is adopted for electricity generation are compared.

In summary, the contributions of this paper are:

- $\quad$ an exploration of the sensitivity of PV production in Riyadh to panel tilt angle

- demonstration of the benefit of optimising the orientation of embedded PV generators to match a load profile that is substantially driven by air conditioning

\section{EQUATIONS OF SOLAR PHOTOVOLTAIC ENERGY SYSTEMS}

Full details of the mathematical model of equations presented in this section can be found in [11], [12]. The average energy flux $S$ that reaches the top of the Earth's atmosphere from the sun is $1.367 \mathrm{~kW} / \mathrm{m}^{2}$ and reaches a benchmark value of approximately $1 \mathrm{~kW} / \mathrm{m}^{2}$ for a clear day by the time it reaches a plane surface on Earth. The model is based on the assumption that the total energy flux is equal to the sum of beam (i.e. direct) radiation and the diffuse radiation (as a result of being scattered by clouds and particles), neglecting the effect of the reflected radiation from the environment (that is, components reflected from nearby buildings, trees, roofs, ground, and so on).

The Earth's tilt angle with respect to the plane of its orbit is $23.45^{\circ}$. The declination of the sun $\delta$ (that is, the angle between the plane containing the Earth's track around the sun and a vector pointed from the Earths Centre) for any given day n where $\mathrm{n}=1$ on the 1 st January is given as follows:

$$
\delta=23.45 \sin \{360(284+n) / 365\}
$$

Let $\varphi$ denote the latitude location of a PV system, then the solar sunrise $\left(-\omega_{s}\right)$ and sunset $\left(\omega_{s}\right)$ times of the day is given as follows:

$$
\omega_{s}=\arccos (-\tan \varphi \tan \delta)
$$

The clear sky energy flux ( $\sin \alpha$ ) for each solar hour $i$ of the day can be computed using the following equation:

$$
\sin \alpha_{i}=\sin \delta \sin \varphi+\cos \delta \cos \varphi \cos \omega_{i}
$$


where $\omega_{i}$ denotes solar hour angle of the $i$ th hour (the time before solar noon expressed as negative degrees $\left(-\omega_{i}\right)$, and the time after solar noon expressed as positive degree $\left(\omega_{i}\right)$ and $\omega_{\mathrm{i}}$ equals $0^{\circ}$ at solar noon. The solar hour of a given location on the Earth denotes the angle between the longitude of that location and the longitude that is parallel to the beam of the sun at that location (that is, for instance, $0^{\circ}, 15^{\circ}, 30^{\circ}$ and $45^{\circ}$ represent 12:00 noon, $1: 00 \mathrm{pm}, 2: 00 \mathrm{pm}$ and 3:00 pm, respectively). The number of sunshine hours for the day s can be computed using the following equation:

$$
s=s_{h} d
$$

where $s_{h}$ is obtained by the subtraction of solar hour angle for sunrise from that of the sunset and $d$ is the clearness factor for the day ( $d=1$ if the day has clear sun, $d=0.75$ if sunny day has some light cloud, $d=0.5$ if sunny day is cloudy, and so on). The clearness index for the day is given by the following equation [6]:

$$
K=e_{c} \sqrt[3]{d}
$$

where $e_{c}$ is an empirical constant defined as $e_{c}=0.65$ if $\varphi>$ $45^{\circ}$ or $e_{c}=0.75$ if $\varphi \leq 45^{\circ}$. The solar beam energy and solar diffuse energy denoted $B$ and $D$ respectively, are given by the following equations:

$$
\begin{aligned}
& B=1.11 H_{o} K^{2} \\
& D=H_{o} K-B
\end{aligned}
$$

where $H_{o}$ is the extra-terrestrial energy directed to a unit horizontal area over a given day and location, and is estimated as:

$$
H_{o}=\sum_{i=1}^{i=N} 1.367 \sin \alpha_{i}
$$

where $N$ is the number of integer hours of daylight hours. Therefore, the total energy (in $\mathrm{kW}$ ) that can be generated by a PV plant of $X$ (in KWp) capacity and inverter efficiency of $\eta$ (in \%) on a particular day can be written as follows:

$$
E_{T}=\sum_{i=1}^{i=24} \eta \times X \times \sin \alpha_{i} \times G_{i}
$$

where

$$
\begin{gathered}
E_{T}=\text { Total energy generated by the plant in a day } \\
\qquad G_{i}=B r_{b_{i}}+D r_{d} \\
r_{b_{i}}=\frac{\cos (\varphi-\beta) \cos \delta \cos \omega_{i}+\sin (\varphi-\beta) \sin \delta}{\cos \varphi \cos \delta \cos \omega_{i}+\sin \varphi \sin \delta} \\
r_{d}=\frac{1}{2}(1+\cos \beta)
\end{gathered}
$$

where $\beta$ is the tilt angle (that is, the angle between the plane of the PV panel surface and horizontal) which when positive means that the surface orientation is towards the equator and when negative means the surface is towards the pole, $r_{b}$ and $r_{d}$ are known as the panel angle incident beam radiation and panel angle incident diffuse radiation factors, respectively.

\section{Optimal Tilt ANGLE Algorithm}

Consider the problem of finding the optimal tilt angle $\beta$ and azimuth angle $\mathrm{z}$, that gives the maximum electrical power from a set of PV generation plants in the region of Riyadh with total capacity of $1000 \mathrm{MWp}$ and inverter efficiency of 98\%, given that it is known that the peak demand for electric power in KSA occurs between 12:00 and 17:00 in summer months. The algorithm to find the optimal $\beta$ and azimuth angle $\mathrm{z}$ takes into account the shape of the demand profile (refer to Fig. 2) by matching the peak demand time with the time of the day when solar energy generation is maximum.

The algorithm is given as follows:

STEP 1: Create a vector of 24 elements - each element being the solar hour angle for each hour of the day.

STEP 2: Create a new vector by offsetting each elements of the vector created in STEP 1 so that solar noon falls at peak time.

STEP 3: For $n=1$ to $n=365$

STEP 4: Compute $\delta$ using the Equation (1)

STEP 5: Compute solar hour angle $\omega_{s}$ for sunrise and sunset using the Equation (2)

STEP 6: Compute the clear sky energy flux $(\sin \alpha)$ for each solar hour $i$ of the day using Equation (3)

STEP 7: Set the clear sky energy flux $(\sin \alpha)$ for each solar hour $i$ of the day outside the daylight hours to zero (daylight hours are the hours between the solar hour angle for sunrise and sunset inclusive).

STEP 8: Compute s - the number of sunshine hours for the day - using the Equation (4)

STEP 9: Compute the clearness index $\mathrm{K}$ for the day using the Equation (5)

STEP 10: Compute the solar beam energy $B$ and the solar diffuse energy $D$ using Equations (6) and (7), respectively.

STEP 11 and STEP 12: Find the optimal $\beta$ for the day by maximizing the total energy generated on the day using Equation (9); the total energy generated on any given day for this can be computed using the following equation:

$$
E_{T}=\sum_{i=1}^{i=24} 0.98 \times 1000000 \times \sin \alpha_{i} \times G_{i}
$$




\section{NUMERICAL RESULTS}

MATLAB software can be used to implement the algorithm given in Section Ш. In particular, MATLAB's nonlinear unconstrained optimization algorithm fminunc can be used for solving the optimization problem.

Fig. 3 shows the typical nature of weather in Riyadh over the months of the year. The climate of Riyadh (latitude $24.63^{\circ}$ ) is typical of the entire Arabian peninsula which experiences mean cloud cover under $15 \%$ with a near to $0 \%$ cloud cover centred on Riyadh $47^{\circ} \mathrm{E}, 25^{\circ} \mathrm{N}$ [9]. Incorporating the normalized daily sunshine data into the algorithm (that is, the clearness index for all the days of January being 7/11, all the days of February being $8 / 11$, all days of March being $7 / 11$, and so on), the optimum average monthly tilt angle is shown in Fig. 4.

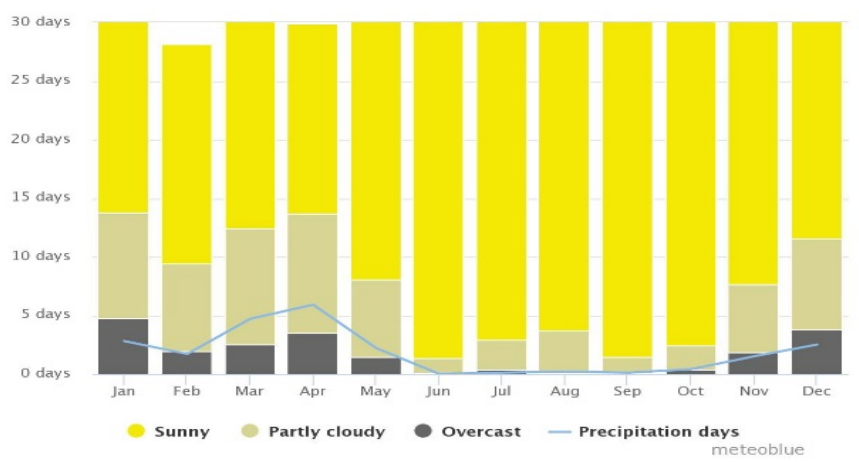

Figure 3: Cloudy, sunny, and precipitation days [15]

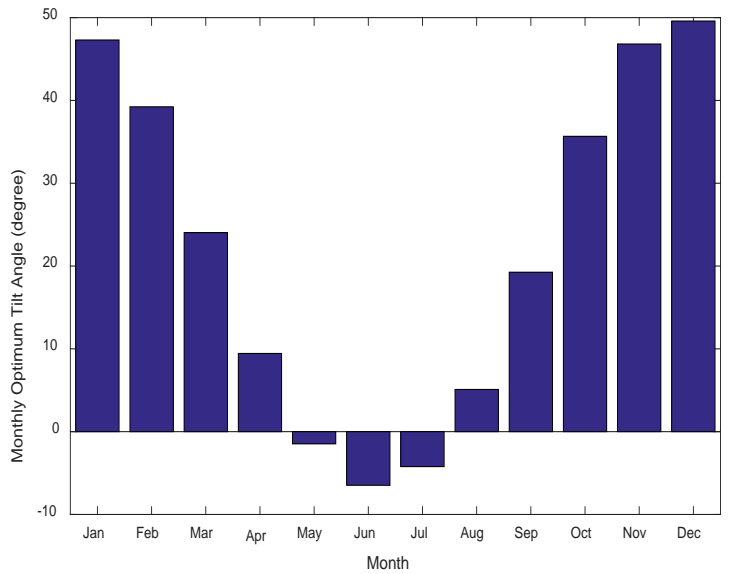

Fig. 4. Riyadh City’s Optimum Monthly Tilt Angle

Negative tilt angles in Fig. 4 correspond to reversal of the panel to be north-facing rather than south facing. However, optimum tilt and azimuth angles we are interested in are those for which the maximum PV generation and the actual demand maximum occur at the same time so that distributed PV generation around the city mitigates the challenging peak demand seen by the large scale conventional power plants. The time at which the actual load curve peaks is $14 \mathrm{hr} 22$ minutes.

The time at which the actual load curve peaks is 14 . 22. The peak of the unmatched PV generation daily curve is at 12 noon. The matching azimuth angle to 14.22 is $35.4285^{\circ}$ and this value has been used to obtain matched results. The plot of the optimal tilt angle computed for each day of the year using the algorithm described in Section $\amalg$ with sunshine hours for Riyadh matched with peak load profile is shown in Fig. 5. To evaluate the possible impact of PV generation on summer demand we assume that distributed PV plants with a total capacity of $1 \mathrm{GWP}$ are deployed around the city. Investigation of the roof and car parking areas of shopping malls, schools, and mosques has shown this is feasible. Fig. 6 shows the solar energy generation of the plant for each day of the year where the optimal tilt angle for each day is used for computing the daily plant electricity generation.

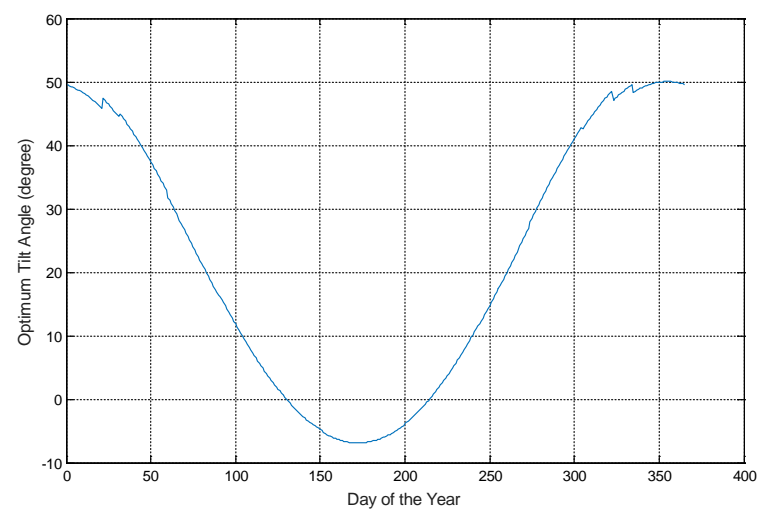

Fig. 5. Optimum Tilt Angle for Each Day of the Year for Riyadh City

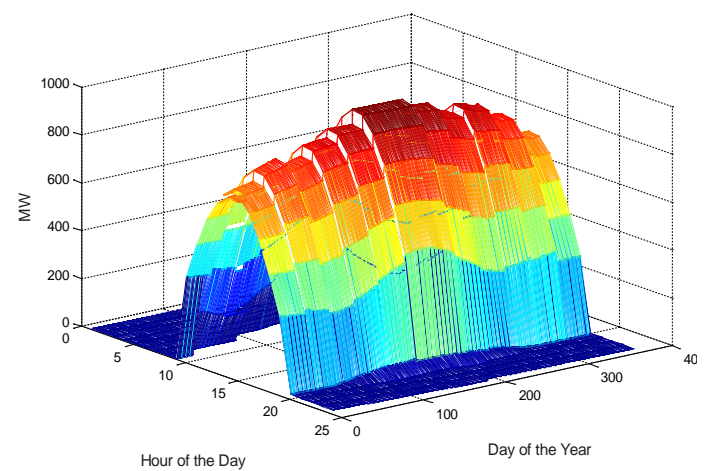

Fig. 6: A Mesh of Monthly PV Generation of a Plant in Riyadh City with Capacity of 1 GWp computed for the Whole Year Using Optimum Tilt Angle for Each Day

Fig. 7 shows the plot of the monthly solar plant electricity generation computed for the whole year with a fixed tilt angle of $2.3830^{\circ}$, which is obtained by taking the average of the optimum tilt angles for five summer months (May to September). This 
result also shows that the maximum power generation in the year is $993.08 \mathrm{MW}$ (the output power achieved under full solar radiation and under set standard test conditions of the installed solar plant) and the tilt angle at which that occurred is $-1.1059^{\circ}$ which is on the $211^{\text {th }}$ day of the year (that is, early-August). Fig. 8 shows the plot of the daily solar plant electricity generation computed for the entire year when the tilt angle is fixed to the value of $-1.1059^{\circ}$.

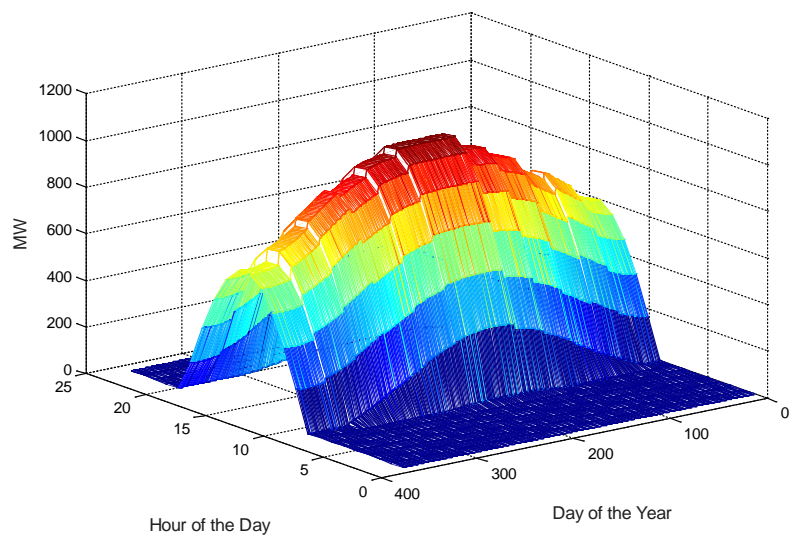

Fig. 7. A Mesh of the Monthly Solar Plant Electricity Generation computed for the Whole Year with a Fixed Tilt Angle of $2.3830^{\circ}$ obtained by taking the average of the tilt angles for five summer months (May to September)

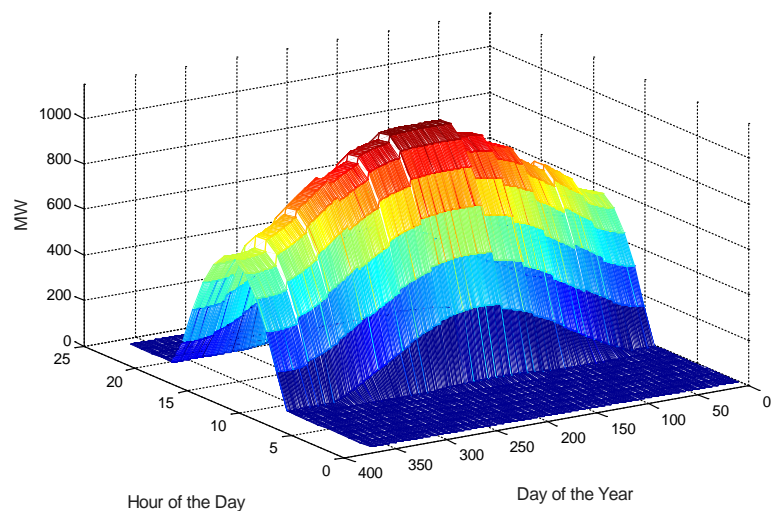

Fig. 8. A Mesh of the Monthly Solar Plant Electricity Generation computed for the Whole Year with a Fixed Tilt Angle of $-1.1059^{\circ}$.

Fig. 9 and 10 show the PV plant generation for selected days of the year (i.e. the $75^{\text {th }}$ day and the $190^{\text {th }}$ day) computed using the optimal value of $\beta$ for each day and the various fixed values of $\beta$.

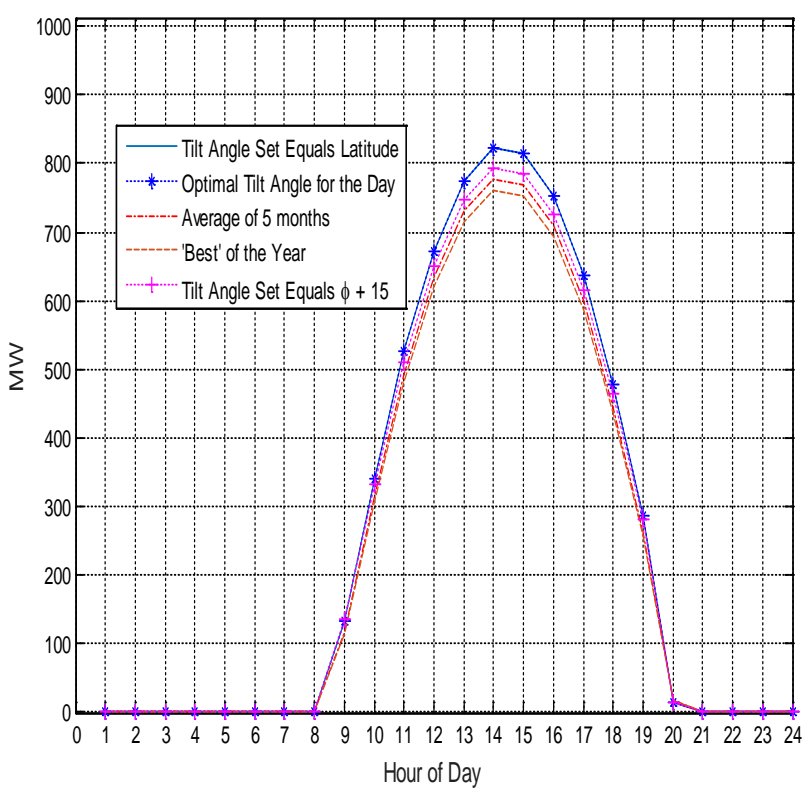

Fig. 9. Different Beta Values on $75^{\text {th }}$ Day of the Year

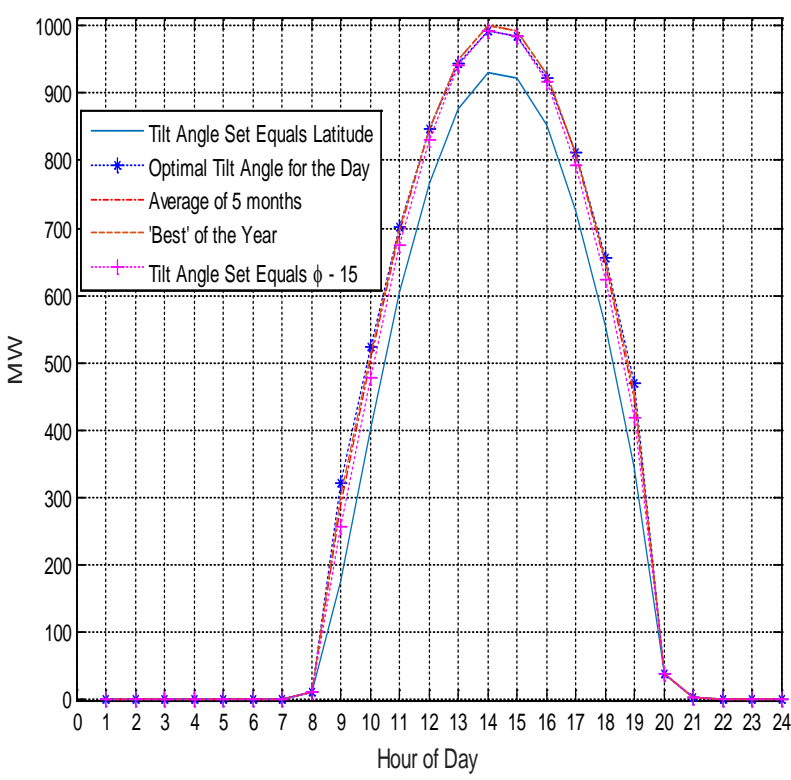

Fig. 10. Maximum PV Plant Generation for Different Beta Values on $190^{\text {th }}$ Day of the Year

The total installed generation capacity of KSA is around 54 GW and the generation units are distributed throughout the major cities. In particular, the total peak load demand for the city of Riyadh is $20.349 \mathrm{GW}$ in the year 2015 [2]. The associated load curves for the matched and unmatched cases when the set of PV generation units of 1,000 MWp capacity that we have considered throughout this paper is installed to supplement the energy demand in that city are shown in Fig. 11. 

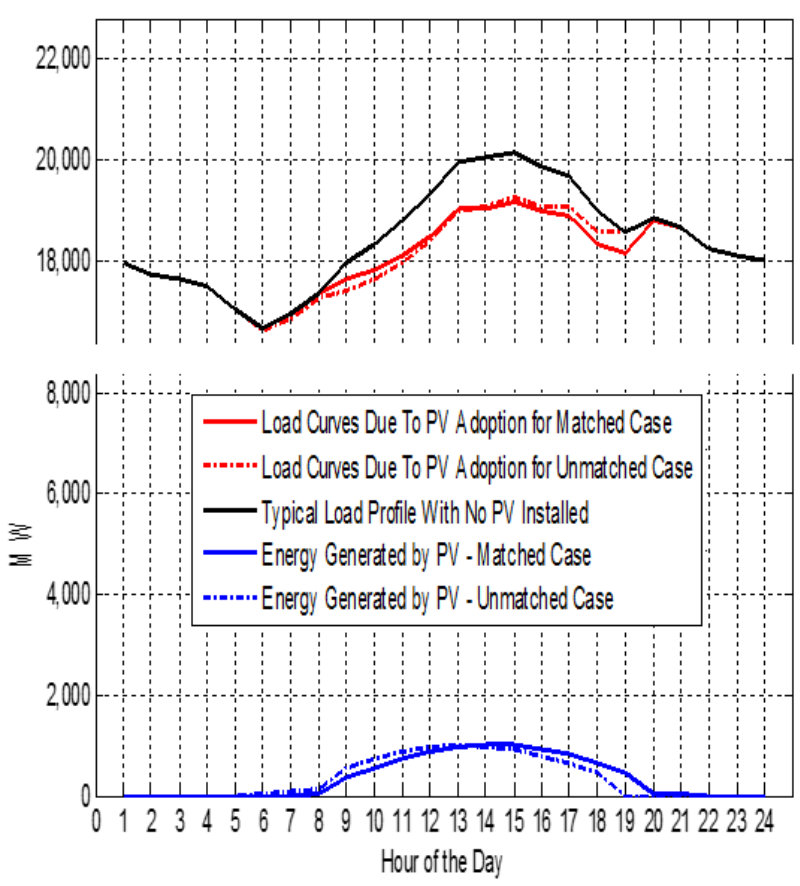

Fig. 11. The load curves for the matched and unmatched cases when the PV generation unit is adopted for electricity generation in a district of Riyadh city.

\section{DISCUSSION}

The present paper has presented an algorithm for finding optimum tilt angle for PV systems matched to the timing of the peak demand on the load profile. The optimum tilt angle for a PV system is obtained by the optimization (maximization) of the amount of total energy that can be generated by the PV system for a given period (such as a day, a calendar month, and a season). The optimum values of PV systems' tilt angle for the city of Riyadh (KSA) were determined using the presented algorithm and the main conclusions that may be drawn from the results are as follows:

- $\quad$ The daily optimum tilt angle varies for each day of the year; for Riyadh, it varies from the minimum value of $-6.90^{\circ}$ on the $172^{\text {th }}$ day of the year to a maximum value of $50.03^{\circ}$ on the $355^{\text {th }}$ day of the year.

- The monthly optimum varies from the minimum value of $-6.48^{\circ}$ in June to a maximum value of $49.59^{\circ}$ in December.

- $\quad$ For addressing the peak load problem due to AC that occurs during the summer, fixing the tilt angle to the value for which yearly maximum energy is obtained throughout the year (that is, $-1.1059^{\circ}$ ) or to the value of average of daily optimum tilt angle of five months throughout the year (that is, $2.3830^{\circ}$ ) will be sufficient to harvest most of the solar radiation as could be achieve by using optimum daily tilt angle during the summer period.

\section{CONCLUSION}

In this paper, a possible way of managing electricity peak demand in KSA is proposed. In order to improve national electricity generation capacity in a practical way, the deployment of PV panels with slope and orientation that are optimized with respect to the timing of the demand profile can be effected. The algorithm to achieve that, as presented in this paper, addresses this problem by obtaining the value of the tilt angle that will satisfy peak demand most effectively. However, the final selection of tilt angle also needs to take account of the effect of dust on PV efficiency, which is significant at this location. Further work is in progress to consider this issue.

\section{REFERENCES}

[1] S. Nachet and M. Aoun, "The saudi electricity sector: Pressing issues and challenges," 2015.

[2] Electricity \& Cogeneration Regulatory Anuthority, "Annual statistical booklet for electricity and seawater desalination industries," ECRA, Riyadh- Saudi Arabia, Tech. Rep. 7, 2014.

[3] H. Jun, "Saudi arabia’s domestic energy situation and policy: Focusing on its power sector," Kyoto bull islam area stud, vol 6, pp. 107-135, 2013.

[4] S. Al-Ajlan, A. Al-Ibrahim, M. Abdulkhaleq and F. Alghamdi, "Developing sustainable energy policies for electrical energy conservation in saudi arabia," Energy Policy, vol 34, no 13, pp. 15561565, 2006.

[5] M. N. Rahman, "Consumer behavior and retail market consumerism in KSA," International journal of scientific \& engineering research, vol 3, no 11, 2012.

[6] F. R. Pazheri, N. H. Malik, A. A. Al-Arainy, S. Ottukulotk, M. F. Othman and E. A. Al-Ammar, "Use of renewable energy sources in saudi arabia through smart grid," Journal of energy and power engineering, vol 6, no 7, pp. 1065, 2012.

[7] S. Rehman, M. A. Bader and S. A. Al-Moallem, "Cost of solar energy generated using PV panels," Renewable and sustainable energy reviews, vol 11, no 8, pp. 1843-1857, 2007.

[8] H. Khorasanizadeh, K. Mohammadi, and A. Mostafaeipour, "Establishing a diffuse solar radiation model for determining the optimum tilt angle of solar surfaces in Tabass, Iran,” Energy Convers. Manag., vol. 78, pp. 805814, 2014.

[9] M. Benghanem, “Optimization of tilt angle for solar panel: Case study for Madinah, Saudi Arabia,” Appl. Energy, vol. 88, no. 4, pp. 1427-1433, 2011.

[10] J. A. Duffie and W. A. Beckman, Solar engineering of thermal processes. John Wiley \& Sons, 2013.

[11] B. Liu and R. Jordan, "Daily insolation on surfaces tilted towards equator,” ASHRAE J.;(United States), vol. 10, 1961.

[12] S. R. Madeti and Z. Ahmad, "Development of a MATLAB / LabVIEW model for optimal tilt angle and maximum power generation of a PV module," in International Conference on Computational Intelligence and Communication Networks, 2015, pp. 1493-1498. 
